\section{GW23-e1483 ELECTRICAL STORM AS THE INITIAL MANIFESTATION OF AN ELDERLY PATIENT WITH ACUTE MYOCARDIAL INFARCTION (ONE CASE REPORT)}

doi:10.1136/heartjnl-2012-302920k.8

Ye Zi, Zhan Hong, Liu Jiang-hui, Li Xin, Liao Xiao-xing, Xiong Yan, Cai Rui-bing, Xu Jia, Zhan Wei, Zhan Hong. Department of Emergency, the First Affiliated Hospital of Sun Yat-Sen University

Objectives To investigate the characteristics and therapeutic method of electrical storm (ES) associated with acute coronary syndrome (ACS).

Methods The clinical data of one elderly patient with ES induced by ACS were collected and relevant literature were reviewed.

Results Case report A 73-year-old man manifested a sudden loss of consciousness when admitting to emergency department due to dizziness. Physical examination showed deep comatose; symmetric pupils with no responding to light at diameter of $4.5 \mathrm{~mm}$; weak breathing and disappearance of main arteries fluctuations. It was ventricular fibrillation (VF) with blood pressure of 0 on electrocardio monitoring. According to his family, the patient has been more than 10 years of hypertension, and BP is controlled at optimal levels. This case of cardiac arrest occurred in the hospital. The onset of the first hour, electric activities displayed repetitive episodes of VF and ventricular tachycardia (VT). Cardiopulmonary cerebral resuscitation (CPCR) was immediately started. At first the patient was successfully converted VF/VT into sinus rhythm 11 times with biphasic waveforms 200J, but the sinus rhythm could not be sustained. We increasing energy gradually, using the biphasic waveforms 300J and 360J defibrillated 2 times respectively. Arrhythmia drugs: amiodarone, lidocaine were administered by intravenous at the same time. $1 \mathrm{~h}$ after admission, ES terminated. Furthermore, temporary pacemaker was installed for ventricular activities weakened, and cooling was initiated by use of external cold blankets and infusion with Ringer's solution $\left(4^{\circ} \mathrm{C}\right)$. After $1.5 \mathrm{~h}$ rescued, patient returned spontaneous circulation, and approximately $4 \mathrm{~h}$ since cardiac arrest, he regained consciousness with intact cerebral function. The results of determinations on admission showed: Troponin T: $0.13 \mathrm{ng} / \mathrm{ml}$, Pro-BNP: $2329 \mathrm{pg} / \mathrm{ml}$. After electric activities stabilising, ECG demonstrated sinus rhythm with ST-segment elevation $0.2-0.4 \mathrm{mV}$ and $\mathrm{T}$ inversion in the percordial leads V2 V6, and echocardiography suggested acute myocardial infarction with EF $68 \%$. Acute extensive anterior myocardial infarction was diagnosed finally. During patient's hospitalisation, IABP was used to assist circulation, and secondary prevention drugs for coronary heart disease were given as the main treatment. The patient discharged from hospital on day 21 with mild renal dysfunction only. During 60 days follow-up, the patient can provide his basic daily life for himself with no chest pain or syncope.
Conclusions ES is one of the major electrophysiology pathogenesis to result in cardiac arrest and SCD. The aetiology of ES varies and myocardial ischaemia, especially ACS, is the most common cause. Patients' condition is severe and prognosis is poor. The characteristics of ES is repeated syncope ${ }^{[1]}$, and electric activities represent with recurrent episodes of VF/VT and are need for defibrillation repeatedly[2]. Electrical cardioversion as soon as possible is the primary measure for stabilising haemodynamics; and interrupting sympathetic activity timely is the most critical strategies in acute phase. The guideline ${ }^{[3]}$ point out that, the only method to control ES is the intravenous $\beta$-blockers. Moreover, select a reasonable opportunity to revascularisation, is considered a fundamental means for treating and preventing ES associated with ACS.

This case may be the first report about electrical storm as the initial manifestation of an elderly patient with acute myocardial infarction. The good neurological outcome and the favourable short-term prognosis during 60-day follow-up of this patient are closely related with high quality CPCR and advanced cardiovascular life support technologies the first time. In addition, the high-energy (300 360J) biphasic waveforms defibrillation in the termination of VT/VF/ES caused by ACS has not been reported either. Once ES diagnosis was confirmed, shall clinical doctors take early high- energy biphasic defibrillation as the first consideration to improve the success rate of cardioversion? And compared with low-energy defibrillation, high- energy will lead to more myocardial damage and complications? All above are worth further exploration. 